\title{
ON THE TOPOLOGY OF SASAKIAN MANIFOLDS
}

\author{
GHEORGHE PITIŞ
}

\begin{abstract}
The notion of $q$-bisectional curvature of a Sasakian manifold $M$ is defined. It is proved that if $M$ has lower bounded $q$-bisectional curvature and contains a compact invariant submanifold tangent to the structure vector field then $M$ is compact. Myers and Frankel type theorems for Sasakian manifolds with lower bounded and positive $q$-bisectional curvature, respectively, are also given.
\end{abstract}

\section{Introduction}

Let $M$ be a complete connected Riemannian manifold. An important theorem, proved by Myers [11], asserts that if $M$ has sectional curvature $\geq k_{0}>0$ (or more generally, if all the eigenvalues of the Ricci tensor are $\geq(\operatorname{dim} M-1) k_{0}>$ 0 ) then $M$ is compact, its diameter is $\leq \pi / \sqrt{k_{0}}$ and has finite fundamental group.

Another remarkable theorem, due to Frankel [2], asserts that if $M$ has positive sectional curvature, then any two compact totally geodesic submanifolds $N$, $P$ of $M$ and such that $\operatorname{dim} N+\operatorname{dim} P \geq \operatorname{dim} M$, must intersect. He also proved that in the case of a complete connected Kähler manifold with positive sectional curvature the same conclusion holds if we replace the hypothesis totally geodesic by analytic. Such results were proved by Goldberg and Kobayashi [5] for Kähler manifolds with positive bisectional curvature. Frankel's theorems were extended by Gray [6] to nearly Kähler manifolds, by Marchiafava [10] to quaternionic Kähler manifolds and by Ornea [12] to locally conformal Kähler manifolds and to Sasakian manifolds in the case when the submanifolds $N$ and $P$ are invariant and tangent to the structure vector field of $M$.

Recently, Kenmotsu and Xia [7], [8] proved Frankel type theorems for Kähler manifolds in the more general case when $M$ has either partially positive sectional curvature or partially positive bisectional curvature.

Our purpose is to give Myers and Frankel type theorems for a Sasakian manifold $M$ under weaker conditions on the curvature of the manifold. The second section of this paper is devoted to the notion of $q$-bisectional curvature for such a manifold. We remark that if $q=1$ then it is exactly the $F$-bisectional

Received June 18, 2001. 
curvature of the manifold [13], [14] and so the class of Sasakian manifolds with positive (or lower bounded) $q$-bisectional curvature is richer than that of Sasakian manifolds with positive (or lower bounded) $F$-bisectional curvature. We prove that if $M$ has lower bounded $q$-bisectional curvature and contains a compact invariant submanifold tangent to the structure vector field of $M$ then $M$ must be compact (Theorem 2.3 and Corollary 2.4). In section 3 we prove a Myers type theorem for Sasakian manifolds with lower bounded $q$-bisectional curvature (Theorem 3.2). The last section is devoted to the proof of a Frankel type theorem for Sasakian manifolds with positive $q$-bisectional curvature and we obtain an extension of Ornea's Theorem 2, [12].

\section{2. q-bisectional curvature}

Let $M$ be a Sasakian manifold and denote by $F, \xi, \eta, g$ its fundamental tensor fields. For any vector fields $X, Y \in \mathscr{X}(M)$, orthogonal to $\xi$, the $F$-bisectional curvature $\mathscr{H}$ of $M$ is defined by

$$
\mathscr{C}(X, Y)=\frac{\mathscr{R}(X, F X, Y, F Y)}{\|X\|^{2}\|Y\|^{2}},
$$

where $\mathscr{R}$ is the Riemann-Christoffel curvature tensor of $M$. Then for any $X^{\prime}, Y^{\prime} \in \mathscr{X}(M)$ such that

$$
\operatorname{span}_{\mathrm{R}}\left\{X^{\prime}, F X^{\prime}\right\}=\operatorname{span}_{\mathrm{R}}\{X, F X\}, \quad \operatorname{span}_{\mathrm{R}}\left\{Y^{\prime}, F Y^{\prime}\right\}=\operatorname{span}_{\mathrm{R}}\{Y, F Y\},
$$

we obtain

$$
\mathscr{H}\left(X^{\prime}, Y^{\prime}\right)=\mathscr{H}(X, Y)
$$

Moreover

Proposition 2.1. For any $X, Y \in \mathscr{X}(M)$, orthogonal to $\xi$, we have

$$
\begin{aligned}
\mathscr{H}(X, Y)=\frac{1}{\|X\|^{2}\|Y\|^{2}}\{\mathscr{R}(X, Y, X, Y)+\mathscr{R}(X, F Y, X, F Y) \\
\left.+2\left[g^{2}(X, Y)-\|X\|^{2}\|Y\|^{2}+g^{2}(X, F Y)\right]\right\} .
\end{aligned}
$$

Proof. By the Lemma, pg. 93, [1], on a contact manifold we have

$$
\begin{aligned}
\mathscr{R}(F Y, Y, X, F X)=\mathscr{R}( & Y, X, X, Y) \\
& +\mathscr{R}(F Y, X, X, F Y)-2 \mathscr{P}(X, Y, X, F Y),
\end{aligned}
$$

where

$$
\begin{aligned}
\mathscr{P}(X, Y, Z, U)=d \eta(X, Z) g(Y, U)-d \eta(X, U) g(Y, Z) \\
-d \eta(Y, Z) g(X, U)+d \eta(Y, U) g(X, Z) .
\end{aligned}
$$


But $M$ is Sasakian, hence $d \eta=\Omega$, with $\Omega(X, Y)=g(X, F Y)$, and then

$$
\mathscr{P}(X, Y, X, F Y)=g^{2}(X, Y)+g^{2}(X, F Y)-\|X\|^{2}\|Y\|^{2} .
$$

Now, from (1) and taking into account (2), (3), we obtain the announced formula.

Let $T_{x} M$ be the tangent space to the Sasakian manifold $M$ at the point $x$ and we denote by $\mathscr{S}=\left\{X_{1}, \ldots, X_{q}\right\} \subset T_{x} M$ an orthonormal system of vectors orthogonal to $\xi$. Then the vectors of the system $F \mathscr{S}=\left\{F X_{1}, \ldots, F X_{q}\right\}$ are orthogonal to $\xi . \mathscr{S}$ is called an $F$-orthonormal $q$-system of tangent vectors at $x$ if $\mathscr{S} \cup F \mathscr{S}$ is orthonormal. We remark that $q \leq\left[\frac{1}{2} \operatorname{dim} M\right]$ and for such a system $\mathscr{S}$ and for any tangent vector $X \in T_{x} M$, orthogonal to $\xi$, we can consider the scalar

$$
\mathscr{H}_{q}(X, \mathscr{S})=\sum_{i=1}^{q} \mathscr{H}\left(X, X_{i}\right) .
$$

Now, taking into account Proposition 2.1, we obtain

Proposition 2.2. Let $X$ be a unit tangent vector at $x \in M$ and $\mathscr{S}$ be an $F$-orthonormal $q$-system at $x$. If $\mathscr{S}^{\prime} \subset T_{x} M$ is an orthonormal system such that $\operatorname{span}_{\mathrm{R}} \mathscr{S}^{\prime}=\operatorname{span}_{\mathrm{R}} \mathscr{S}$ then:

a) $\mathscr{S}^{\prime}$ is an F-orthonormal q-system

b) $\mathscr{H}_{q}\left(X, \mathscr{S}^{\prime}\right)=\mathscr{H}_{q}(X, \mathscr{S})$.

From Proposition 2.2 it follows that $\mathscr{H}_{q}(X, \mathscr{S})$ is depending only on the subspace of $T_{x} M$ spanned by $\mathscr{S}$, but not on the $F$-orthonormal $q$-system $\mathscr{S}$. We call $\mathscr{H}_{q}(X, \mathscr{S})$ the $q$-bisectional curvature of $M$ at the point $x$ and we remark that for $q=1$ it is exactly the $F$-bisectional curvature of $M$.

In the following of this section we shall construct $F$-orthonormal systems and these will be used in order to give information about the topology of the manifold.

Let $N$ be a $2 r$-dimensional $(r \geq 1)$ submanifold of the complete connected Sasakian manifold $M$ and we assume $N$ to be invariant (i.e. $F T_{x} N \subseteq T_{x} N$ for any $x \in N)$ and it is tangent to $\xi$. If $\left\{e_{1}, \ldots, e_{r}, F e_{1}, \ldots, F e_{r}, \xi\right\}$ is an adapted basis of $T_{x} N$ then $\mathscr{B}=\left\{e_{1}, \ldots, e_{r}\right\}$ is, obviously, an $F$-orthonormal $r$-system. Moreover, if $\gamma:[0, \infty) \rightarrow M$ is the geodesic starting from $x$ and orthogonal to $N$ at $x$ then the system $\tilde{\mathscr{B}}$, obtained from $\mathscr{B}$ by parallel translation along $\gamma$ is an $F$-orthogonal $r$-system, too. Indeed, if $E_{i}$ is obtained by parallel translation of $e_{i}$ along $\gamma$ then we have

$$
\nabla_{\gamma^{\prime}} E_{i}=0, \quad E_{i}(\gamma(t))=E_{i}(t)=e_{i},
$$


hence $E_{i}$ is normal to $\gamma$. Similar equalities hold for the vector fields $\tilde{E}_{i}$, obtained from $F e_{i}$ by parallel translation along $\gamma$, and therefore $\tilde{E}_{i}$ are normal to $\gamma$, too. But by using the well-known equality, true on a Sasakian manifold, ([1], Theorem, pg. 73)

$$
\left(\nabla_{X} F\right) Y=g(X, Y) \xi-\eta(Y) X,
$$

we have

$$
\nabla_{\gamma^{\prime}}\left(F E_{i}\right)=0,
$$

and because $F E_{i}(\gamma(t))=F e_{i}$, it follows $\tilde{E}_{i}=F E_{i}$, which proves that $\tilde{\mathscr{B}}$ is an $F$-orthonormal $r$-system.

THEOREM 2.3. Let $M$ be a complete connected Sasakian manifold of dimension $2 n+1 \geq 5$. If for some $r \geq 1$ there exists a $2 r+1$-dimensional compact invariant submanifold $N$, tangent to $\xi$ and such that

$$
\liminf _{t \rightarrow \infty} \int_{0}^{t} \mathscr{H}_{r}\left(\gamma^{\prime}(s), \tilde{\mathscr{B}}\right) d s>0,
$$

for any $x \in N$ and for any $F$-orthonormal $r$-system $\mathscr{B}$ of $T_{x} M$ then the manifold $M$ is compact.

For the proof of this theorem we drew one's inspiration from [8].

Proof. If $M$ is not compact then, by Theorem 1 of [3], there exists $x \in N$ and a geodesic $\gamma:[0, \infty) \rightarrow M$, orthogonal to $N$ at $x$, and such that

$$
\operatorname{distance}(\gamma(t), N)=\text { length } \gamma_{[[0, t]},
$$

hence $\gamma$ has no conjugate points. By putting $\mathscr{H}(t)=\frac{1}{2 r} \mathscr{H}_{r}\left(\gamma^{\prime}(t), \tilde{\mathscr{B}}\right)$ and taking into account (4), from [15] (see also [8]) it follows that the scalar Jacobi equation

$$
f^{\prime \prime}+\mathscr{H}(t) f=0
$$

has a solution $\Phi:[0, \infty) \rightarrow R$, satisfying the conditions $\Phi(0)=1, \Phi^{\prime}(0)=0$ and $\Phi\left(t_{0}\right)=0$ for some $t_{0}>0$.

In the following we shall use the well-known expressions of the index form $I$ of the geodesic $\gamma$ along $\gamma_{\mid[a, b]}$ (see [9], t. II, Theorems 5.4 and 5.5, pg. 81)

$$
\begin{aligned}
I_{a}^{b}(X, Y)= & \int_{a}^{b}\left[g\left(X^{\prime}, Y^{\prime}\right)-\mathscr{R}\left(X, \gamma^{\prime}, Y, \gamma^{\prime}\right)\right] d t \\
= & g\left(X^{\prime}, Y\right)(b)-g\left(X^{\prime}, Y\right)(a) \\
& \quad-\int_{a}^{b}\left[g\left(X^{\prime \prime}, Y\right)+\mathscr{R}\left(X, \gamma^{\prime}, Y, \gamma^{\prime}\right)\right] d t
\end{aligned}
$$


for all vector fields $X$ and $Y$ along $\gamma$. Indeed, we consider it for the vector fields $X_{i}, Y_{i}$, defined along $\gamma_{\mid\left[0, t_{0}\right]}$, by

$$
X_{i}(t)=\Phi(t) E_{i}(t), \quad Y_{i}(t)=\Phi(t) \tilde{E}_{i}(t)
$$

They are tangent to $N$ at $\gamma(0)$ and $X_{i}\left(t_{0}\right)=Y_{i}\left(t_{0}\right)=0$. Moreover we have

$$
X_{i}^{\prime}=\Phi^{\prime} E_{i}, \quad Y_{i}^{\prime}=\Phi^{\prime} \tilde{E}_{i}
$$

hence $X_{i}^{\prime}(0)=Y_{i}^{\prime}(0)=0$ and $X_{i}^{\prime \prime}=\Phi^{\prime \prime} E_{i}, Y_{i}^{\prime \prime}=\Phi^{\prime \prime} \tilde{E}_{i}$. Then we have

$$
\begin{gathered}
g\left(X_{i}^{\prime \prime}, X_{i}\right)+\mathscr{R}\left(X_{i}, \gamma^{\prime}, X_{i}, \gamma^{\prime}\right)=\Phi^{\prime \prime} \Phi+\Phi^{2} \mathscr{R}\left(E_{i}, \gamma^{\prime}, E_{i}, \gamma^{\prime}\right) \\
g\left(Y_{i}^{\prime \prime}, Y_{i}\right)+\mathscr{R}\left(Y_{i}, \gamma^{\prime}, Y_{i}, \gamma^{\prime}\right)=\Phi^{\prime \prime} \Phi+\Phi^{2} \mathscr{R}\left(\tilde{E}_{i}, \gamma^{\prime}, \tilde{E}_{i}, \gamma^{\prime}\right)
\end{gathered}
$$

By using Gauss formula we have

$$
g\left(X^{\prime}, Y\right)=g\left(h(X, Y), \gamma^{\prime}\right)
$$

for all $X, Y$ tangent to $N$ and normal to $\gamma$. But $N$ is invariant and tangent to $\xi$ and then we have

$$
h\left(F e_{i}, F e_{i}\right)=-h\left(e_{i}, e_{i}\right) .
$$

Now, from (5), (8), (9) and (10) we give

$$
\begin{aligned}
\sum_{i=1}^{r}\left[I_{0}^{t_{0}}\left(X_{i}, X_{i}\right)+I_{0}^{t_{0}}\left(Y_{i}, Y_{i}\right)\right]=-2 r \int_{0}^{t_{0}} \Phi\left[\Phi^{\prime \prime}+\mathscr{H}(t) \Phi\right] d t \\
-2 r \int_{0}^{t_{0}}\left\|\gamma^{\prime}\right\|^{2} d t \\
=-2 r \int_{0}^{t_{0}}\left\|\gamma^{\prime}\right\|^{2} d t<0,
\end{aligned}
$$

hence $I_{0}^{t_{0}}\left(X_{i}, X_{i}\right)<0$ or $I_{0}^{t_{0}}\left(Y_{i}, Y_{i}\right)<0$ for some $i \in\{1,2, \ldots, r\}$ and therefore $\gamma_{\left[\left[0, t_{0}\right]\right.}$ has a conjugate point. But this contradicts the hypothesis that $\gamma$ has no conjugate points.

We say that $M$ has lower bounded $q$-bisectional curvature at the point $x \in M$ if there exists $k_{0} \in \mathrm{R}$ such that $\mathscr{H}_{q}(X, \mathscr{S}) \geq k_{0}$ for any unit tangent vector $X \in T_{x} M$ and for any $F$-orthonormal $q$-system $\mathscr{S}$. If $k_{0}=0$ then we say that $M$ has nonnegative $q$-bisectional curvature and taking into account (1) we remark that if $M$ has nonnegative $F$-bisectional curvature then its $q$ bisectional curvature is also nonnegative for any $q \leq\left[\frac{1}{2} \operatorname{dim} M\right]$. Hence the family of Sasakian manifolds with nonnegative $q$-bisectional curvature is richer 
than the one containing all Sasakian manifolds with nonnegative $F$-bisectional curvature.

By using the above notions, from Theorem 2.3 we deduce

Corollary 2.4. Let $M$ be a complete connected Sasakian manifold with positive lower bounded $r$-bisectional curvature, $r \leq\left[\frac{1}{2} \operatorname{dim} M\right]$. If $M$ contains a $2 r+1$-dimensional compact invariant submanifold, tangent to $\xi$, then $M$ is compact.

\section{A Myers type theorem}

Let $M$ be a $2 n+1$-dimensional Sasakian manifold and we denote by $M^{*}$ its universal covering space. It is well-known (see for instance [9], t. I, pg. 162) that on $M^{*}$ there is a Riemannian metric $g^{*}$ such that the projection $\pi: M^{*} \rightarrow M$ is an isometric immersion and we define the 1 -form $\eta^{*}$ on $M^{*}$ by $\eta_{p}^{*}=\pi_{p}^{*} \eta_{\pi(p)}$, where $\pi_{p}^{*}$ is the codifferential of $\pi$ at the point $p \in M^{*}$. If $\xi^{* *}$ is its dual vector field with respect to $g^{*}$, i.e. the only vector field satisfying

$$
\eta^{*}\left(X^{*}\right)=g^{*}\left(X^{*}, \xi^{* *}\right)
$$

for any $X^{*} \in \mathscr{X}\left(M^{*}\right)$, then $\xi^{* *}$ is nowhere zero, hence we can consider its associated unit vector field $\xi^{*}$ and we have $\pi_{*, p} \xi_{p}^{*}=\xi_{\pi(p)}$, where $\pi_{*, p}$ is the differential of $\pi$.

Let $\nabla^{*}$ be the Levi-Civita connection on $M^{*}$, associated with the metric $g^{*}$. Then we can define the morphism $F^{*}: \mathscr{X}\left(M^{*}\right) \rightarrow \mathscr{X}\left(M^{*}\right)$ by

$$
F^{*} X^{*}=-\nabla_{X^{*}}^{*} \xi^{*}
$$

for any $X^{*} \in \mathscr{X}\left(M^{*}\right)$ and a straightforward computation shows that the tensor fields $F^{*}, \xi^{*}, \eta^{*}, g^{*}$ define a Sasakian structure on $M^{*}$ (see for instance [1], Theorem, pg. 73).

If $\mathscr{S}=\left\{e_{1}, \ldots, e_{q}\right\}$ is an $F$-orthonormal $q$-system of local vector fields in $M$ then we consider the 1 -forms $\omega_{1}, \ldots, \omega_{2 q}$, defined by

$$
\omega_{i}(X)=g\left(e_{i}, X\right), \quad \omega_{q+i}(X)=g\left(F e_{i}, X\right)
$$

for any $X \in \mathscr{X}(M)$ and $i \in\{1, \ldots, q\}$. We obtain $2 q$ local 1 -forms $\omega_{1}^{*}, \ldots, \omega_{2 q}^{*}$, defined by

$$
\omega_{j, p}^{*}=\pi_{p}^{*} \omega_{j, \pi(p)}
$$

for $j \in\{1, \ldots, 2 q\}$. Their dual local vector fields $e_{1}^{*}, \ldots, e_{2 q}^{*}$, given by formulae similar to (11), satisfy

$$
g\left(\pi_{*} e_{j}^{*}, \pi_{*} Y^{*}\right)=\left(\pi^{*} \omega_{j}\right)\left(Y^{*}\right)=\omega_{j}\left(\pi_{*} Y^{*}\right)=g\left(e_{j}, \pi_{*} Y^{*}\right),
$$


and taking into account $\pi_{*}$ is injective, we deduce $e_{j}=\pi_{*} e_{j}^{*}$. Hence $\mathscr{S}^{*}=$ $\left\{e_{1}^{*}, \ldots, e_{q}^{*}\right\}$ is an $F$-orthonormal $q$-system of local vector fields in $M^{*}$ and by a straightforward computation we deduce

Proposition 3.1. Let $M$ be a Sasakian manifold and $M^{*}$ its universal covering. If $\mathscr{H}_{q}, \mathscr{H}_{q}^{*}$ are the $q$-bisectional curvatures of $M$ and $M^{*}$ respectively, then

$$
\mathscr{H}_{q}^{*}\left(X^{*}, \mathscr{S}^{*}\right)=\mathscr{H}_{q}\left(\pi_{*} X^{*}, \mathscr{S}\right),
$$

for any unit vector field $X^{*} \in \mathscr{X}\left(M^{*}\right)$ and for any $F$-orthonormal $q$-system $\mathscr{S}$ of $M$.

Now we shall prove a Myers type theorem for Sasakian manifolds, namely

TheOREm 3.2. Let $M$ be a complete connected Sasakian manifold with lower bounded q-bisectional curvature $\mathscr{H}_{q} \geq k_{0}>0$. Then:

a) $M$ is compact;

b) the diameter of $M$ is at most equal to $\pi \sqrt{\frac{2 q}{2 q+k_{0}}}$;

c) $M$ has finite fundamental group.

Proof. For two arbitrary points $x$ and $y$ of $M$ denote by $\gamma:[a, b] \rightarrow M$ the minimizing geodesic joining $x$ to $y$. We assume that $\gamma$ is parametrized by its arc length $s$ and $b$ is such that $\gamma(b)$ is the first conjugate point of $\gamma$. If $\mathscr{S}=\left\{e_{1}, \ldots, e_{q}\right\} \subset T_{x} M$ is an $F$-orthonormal $q$-system normal to $\gamma$ at $\gamma(a)$ then, as above, by parallel translation of $\mathscr{S}$ along $\gamma$, we obtain another $F$-orthonormal $q$-system $\tilde{\mathscr{S}}=\left\{E_{1}, \ldots, E_{q}\right\}$ of vector fields defined along $\gamma$ and normal to $\gamma$.

Now, if $\Phi:[a, b] \rightarrow \mathrm{R}$ is a nonzero differentiable function such that $\Phi(a)=\Phi(c)=0$ for some $c \in(a, b)$, then definitions similar to (6) give the vector fields $X_{i}, Y_{i}$ along $\gamma$ and by using (7) we obtain

$$
\begin{aligned}
& \sum_{i=1}^{q}\left[I_{a}^{c}\left(X_{i}, X_{i}\right)+I_{a}^{c}\left(Y_{i}, Y_{i}\right)\right] \\
= & \int_{a}^{c}\left\{2 q \Phi^{\prime 2}-\Phi^{2} \sum_{i=1}^{q}\left[\mathscr{R}\left(E_{i}, \gamma^{\prime}, E_{i}, \gamma^{\prime}\right)+\mathscr{R}\left(F E_{i}, \gamma^{\prime}, F E_{i}, \gamma^{\prime}\right)\right]\right\} d s .
\end{aligned}
$$

But $\gamma_{[[a, c]}$ has no conjugate points and taking into account Proposition 2.1, from (12) we deduce

$$
0<\int_{a}^{c}\left\{2 q \Phi^{\prime 2}-\Phi^{2}\left[\mathscr{H}_{q}\left(\gamma^{\prime}, \tilde{\mathscr{S}}\right)+2 q\right]\right\} d s \leq \int_{a}^{c}\left[2 q \Phi^{\prime 2}-\left(k_{0}+2 q\right) \Phi^{2}\right] d s
$$


For $\Phi(s)=\sin \pi \frac{s-a}{c-a}$, from the above inequalities we obtain

$$
(c-a)^{2}<\frac{2 q}{2 q+k_{0}} \pi^{2} .
$$

But $c$ is arbitrary in $(a, b)$, hence

$$
b-a \leq \pi \sqrt{\frac{2 q}{2 q+k_{0}}}
$$

and because $\gamma$ is parametrized by its arc length, it follows

$$
\operatorname{distance}(x, y)=\operatorname{distance}(\gamma(a), \gamma(b)) \leq \pi \sqrt{\frac{2 q}{2 q+k_{0}}} .
$$

Thus $\mathrm{b}$ ) is proved. From (13) we also deduce that $M$ is bounded and because it is complete, a) is proved too (see [9], t. I, Theorem 4.1, pg. 172). Now, because $M$ is compact, it is well-known that $M^{*}$ is complete and connected ([9], t. I, pg. 76) and taking into account Proposition 3.1, it follows that $M^{*}$ satisfies the hypothesis of Theorem 3.2. Hence, by a) it follows that $M^{*}$ is compact and then the fundamental group of $M$ is finite.

\section{A Frankel type theorem}

THEOREM 4.1. Let $M$ be a complete connected Sasakian manifold with positive $q$-bisectional curvature. If $N$ and $P$ are two compact invariant submanifolds of $M$, tangent to $\xi$ and such that $\operatorname{dim} N+\operatorname{dim} P \geq \operatorname{dim} M+2 q-1$, then $N \cap P \neq \varnothing$.

Proof. If $N \cap P=\emptyset$ then there is a geodesic $\gamma:[0, l] \rightarrow M$, parametrized by the arc length, joining two points $x_{0} \in N, y_{0} \in P$ and realizing the minimum of the distance between $N$ and $P$. We denote by $V_{y_{0}}$ the subspace of $T_{y_{0}} M$, obtained by parallel translation of $T_{x_{0}} N$ along $\gamma$ at the point $y_{0}$. From the hypothesis concerning the dimensions and because $N$ and $P$ are tangent to $\xi$, it follows that $\operatorname{dim}\left(V_{y_{0}} \cap T_{y_{0}} P\right) \geq 2 q$. But $N$ and $P$ are invariant, hence $V_{y_{0}} \cap T_{y_{0}} P$ is invariant under $F$ and because $\xi_{y_{0}} \in V_{y_{0}} \cap T_{y_{0}} P$, it follows that $\operatorname{dim}\left(V_{y_{0}} \cap T_{y_{0}} P\right) \geq 2 q+1$. Moreover, we can find an $F$-orthonormal $q$-system $\mathscr{S}_{y_{0}}=\left\{e_{1}, \ldots, e_{q}\right\} \subset V_{y_{0}} \cap T_{y_{0}} P$. By parallel translation of $\mathscr{S}_{y_{0}}$ along $\gamma$, we obtain a system of $q$ unit vector fields $\tilde{\mathscr{S}}=\left\{E_{1}, \ldots, E_{q}\right\}$, defined along $\gamma$ and such that $a$ vectors of $\tilde{\mathscr{S}} \cup F \tilde{\mathscr{S}}$ are from $\tilde{\mathscr{S}}$ and $b(a+b=2 q)$ of them are from $F \tilde{\mathscr{S}}$.

If $a \geq q$ then we can assume that these vector fields are $E_{1}, \ldots, E_{q}$ and because $P$ is invariant, it follows that $F E_{1}, \ldots, F E_{q}$ are tangent to $P$ at $y_{0}$, 
too. The same argument used if $b \geq q$ shows that always we can find an $F$-orthonormal $q$-system $\tilde{\mathscr{S}}$ of vector fields along $\gamma$, tangent to $N$ at $x_{0}$ and tangent to $P$ at $y_{0}$. Then, by a computation similar to the one used in [8] (the proof of Theorem 3.2.) and taking into account (10), we obtain

$$
\begin{aligned}
\sum_{i=1}^{q}\left[I_{0}^{l}\left(E_{i}, E_{i}\right)\right. & \left.+I_{0}^{l}\left(F E_{i}, F E_{i}\right)\right] \\
& =-\int_{0}^{l} \sum_{i=1}^{q}\left[\mathscr{R}\left(E_{i}, \gamma^{\prime}, E_{i}, \gamma^{\prime}\right)-\mathscr{R}\left(F E_{i}, \gamma^{\prime}, F E_{i}, \gamma^{\prime}\right)\right] d s .
\end{aligned}
$$

Now, by using Proposition 2.1 and taking into account

$$
\sum_{i=1}^{q}\left[g^{2}\left(\gamma^{\prime}, E_{i}\right)+g^{2}\left(\gamma^{\prime}, F E_{i}\right)\right] \leq\left\|\gamma^{\prime}\right\|^{2}=1
$$

from (14) it follows

$$
\begin{aligned}
& \sum_{i=1}^{q}\left[I_{0}^{l}\left(E_{i}, E_{i}\right)+I_{0}^{l}\left(F E_{i}, F E_{i}\right)\right] \\
& \quad=-q l+\int_{0}^{l} \sum_{i=1}^{q}\left[g^{2}\left(\gamma^{\prime}, E_{i}\right)+g^{2}\left(\gamma^{\prime}, F E_{i}\right)\right] d s-\int_{0}^{l} \mathscr{H}_{q}\left(\gamma^{\prime}, \tilde{\mathscr{S}}\right) d s \\
& \quad \leq l(1-q)-\int_{0}^{l} \mathscr{H}_{q}\left(\gamma^{\prime}, \tilde{\mathscr{S}}\right) d s<0
\end{aligned}
$$

We deduce that $I_{0}^{l}\left(E_{i}, E_{i}\right)<0$ or $I_{0}^{l}\left(F E_{i}, F E_{i}\right)<0$ for some $i \in\{1, \ldots, q\}$, contradicting the hypothesis that $\gamma$ has minimal length. Hence $N$ and $P$ must have nonempty intersection.

\section{REFERENCES}

1. Blair, D., Contact manifolds in Riemannian geometry, Lecture Notes in Math. 509 (1976).

2. Frankel, T., Manifolds with positive curvature, Pacific J. Math. 11 (1961), 165-171.

3. Galloway, G., Some results on the occurences of compact minimal submanifolds, Manuscripta Math. 25 (1981), 209-219.

4. Galloway, G., and Rodriguez, L., Intersections of minimal submanifolds, Geom. Dedicata 39 (1991), 29-42.

5. Goldberg, S. I., and Kobayashi, S., Holomorphic bisectional curvature, J. Differential Geom. 1 (1966), 225-233.

6. Gray, A., Nearly Kähler manifolds, J. Differential Geom. 4 (1970), 283-309.

7. Kenmotsu, K., and Xia, C., Intersections of minimal submanifolds in manifolds of partially positive curvature, Kodai Math. J. 18 (1995), 242-249. 
8. Kenmotsu, K., and Xia, C., Hadamard-Frankel type theorems for manifolds with partially positive curvature, Pacific J. Math. 176 (1996), 129-139.

9. Kobayashi, S., and Nomizu, K., Foundations of Differential Geometry, t. I (1963), t. II (1969), Interscience, New-York.

10. Marchiafava, S., Su alcune sottovarieta che ha interesse considerare in una varieta Kaehleriana quaternionale, Rend. Mat. 10 (1990), 493-529.

11. Myers, S. B., Riemannian manifolds in the large, Duke Math. J. 1 (1935), 39-49.

12. Ornea, L., A theorem on nonnegatively curved locally conformal Kaehler manifolds, Rend. Mat. 12 (1992), 257-262.

13. Pitiş, Gh., Rizza's conjecture concerning the bisectional curvature, Riv. Mat. Univ. Parma 16 (1990), 195-203.

14. Rizza, G. B., On the bisectional curvature of a Riemannian manifold, Simon Stevin 61 (1987), 147-155.

15. Tipler, F. J., General relativity and conjugate ordinary differential equations, J. Differential Equations 30 (1978), 165-174.

16. Wu, H., Manifolds of partially positive curvature, Indiana Univ. Math. J. 36 (1987), 525-548.

DEPARTMENT OF EQUATIONS

FACULTY OF MATHEMATICS AND INFORMATICS

UNIVERSITY TRANSILVANIA OF BRAŞOV

2200 BRAȘOV

ROMANIA

E-mail: gh.pitis@info.unitbv.ro 